\section{Rabbit nutrition part I: the good, the bad and the ugly}

\section{Jo Hinde}

Correct nutrition is the cornerstone of rabbit health and it is vital that we understand fully how it can affect our patients, both at home and in the clinic. There is a direct correlation between nutrition and welfare which will be explored and explained.

We will start with a recap of anatomy and physiology including an in-depth look into the rabbit's digestive system, faecal identification and the relevance of caecotrophy. Rabbits are hindgut fermenters and, as such, have specific nutritional needs that differ significantly from those of cats and dogs. They are selective and frequent feeders which means they should spend a significant portion of their day (and evening) searching for food. If they do not have access to this type of feeding or grazing, then this can cause some significant physical and mental health problems.

Nutritional needs will be covered along with delving deeper into the types of commercial foods that are available and why a life-stage diet is important. Rabbits' diets need to be adapted for their current age, health status and living conditions. The lecture will discuss each life stage in depth and provide the current recommended feeding guidelines. These guidelines are based on upto-date information from rabbit specialists and the Rabbit Welfare Association. The importance of hay will be highlighted and the part it plays in digestive, dental and mental health. There is a huge variety of hay, grass and forage that is suitable to feed to rabbits on a daily basis and we will explore the most common ones.

The problems with concentrate foods, especially muesli mix, will be highlighted, along with the range of vegetables and fruit that can be fed, including the pros and cons of each. Treats are a human concept and there will be discussion around this topic, including what commercial treats are readily available and which ones can be dangerous to the rabbit's health and why. This will lead on to highlighting the healthier and safer treat alternatives.

Finally the lecture will show examples of rabbits that are fed correctly and highlight ideal body condition scores, healthy teeth and other examples of the good consequences that arise from providing a well balanced diet.

\section{KEY LEARNING OBJECTIVES}

- Have a greater understanding of a rabbit's digestive anatomy

- Understand what a balanced diet is for each life stage

- Know and be able to recommend optimal dietary advice to owners

\section{MULTIPLE CHOICE QUESTIONS}

1. What is the minimum amount of hay a rabbit should eat daily?
(A) A ball the same size as their head
(B) A ball the same size as their body
(C) A ball the same size as their ear
(D) A ball the same size as their tail

2. What is the percentage of volatile fatty acids ingested in caecotrophs?
(A) $20 \%$
(B) $30 \%$
(C) $40 \%$
(D) $50 \%$

3. At what life stage should alfalfa hay be the main hay source?
(A) Junior
(B) Adult
(C) Mature
(D) All of them

\section{Rabbit nutrition part II: associated problems including anaesthetic considerations}

\section{Jo Hinde}

This lecture will explore rabbit nutrition and the consequences of an unbalanced diet. The types of problems that are commonly linked to poor diets will be explained as well as looking into treatment plans and preventative options.
First, obesity will be discussed. Many of the UK's pets are now overweight and this is of rising concern. All rabbits should be body condition scored every time they visit the veterinary clinic and I also recommend that you teach owners how to do this so that they can add it into their weekly home checks. An overweight rabbit is at risk of developing a range of serious conditions, including but not limited to, gastrointestinal stasis, myiasis, pododermatitis, skeletal problems, cardiac problems and bladder sludge. They will also be a higher general anaesthetic risk.

Feeding a diet that is too rich, contains too much starch, sugar and/or protein can often result in dysbiosis This can easily result in the rabbit being caked in faeces, increasing the risk of bacterial skin infections and sores, and can lead on to the rabbit being more susceptible to myiasis. The microbiome in the caecum is complex and 